\title{
New Nonlocal Charges in SUSY Integrable Models
}

\author{
Ashok Das \\ Department of Physics and Astronomy, \\ University of Rochester, \\ Rochester, NY 14627-0171 \\ USA \\ and \\ Ziemowit Popowicz \\ Institute of Theoretical Physics, \\ University of Wroclaw, \\ 50-205 Wroclaw \\ Poland.
}

\begin{abstract}
In this letter, we study systematically the general properties of the $B$-extension of any integrable model. In addition to discussing the general properties of Hamiltonians, Hamiltonian structures etc, we also clarify the origin of "exotic" charges in such models. We show that, in such models, there exist at least two sets of non-local conserved charges (and more if $N>$ 1 supersymmetry is present) and that the "exotic" charges are part of this non-local charge hierarchy. The construction of these non-local charges from the Lax operator is explained.
\end{abstract}




\section{Introduction:}

Integrable models [1] appear naturally in the study of strings in the matrix model approach. Thus, while the KdV hierarchy is obtained in the double scaling limit of the one matrix model [2], the supersymmetric matrix models lead to a particular supersymmetric version of the KdV hierarchy known as the $N=1$ supersymmetric KdV-B hierarchy [3]. In simple terms, if $u$ denotes the dynamical variable of the $\mathrm{KdV}$ equation

$$
u_{t}=u_{x x x}+6 u u_{x}
$$

where the subscripts represent differentiation with respect to the corresponding variables, then, the $N=1$ supersymmetric KdV-B hierarchy is given by

$$
\Phi_{t}=\Phi_{x x x}+3\left(D(D \Phi)^{2}\right)
$$

Here, $\Phi(x, \theta)=\psi(x)+\theta u(x)$ represents the dynamical variable which is a $N=1$ fermionic superfield with $\theta$ denoting the Grassmann coordinate and

$$
D=\frac{\partial}{\partial \theta}+\theta \frac{\partial}{\partial x}
$$

There are, of course, other supersymmetrizations of the KdV hierarchy that are integrable [4], but it is this particular supersymmetrization [5] that manifests in the study of string theories. Therefore, in this letter, we undertake a systematic study of the properties of such a supersymmetrization. In particular, we show, in section $\mathbf{2}$, that this particular method of supersymmetrization can be applied to any integrable model, although the original study involved the bosonic KdV hierarchy. In section 3, we bring out some general properties of such models, such as the Hamiltonians, Hamiltonian structures, recursion operators etc. In these models, there arise local conserved charges which have opposite Grassmann parity relative to the Hamiltonians of the system. The origin of such "exotic" charges [5] is explained in section 4 , where we identify that such local charges belong to the hierarchy of an infinite set of non-local charges. In fact, we show that, in such models, there exist, at least, two infinite sets of non-local charges and may be more. Explicitly, in the $N=2$ supersymmetric $\mathrm{KdV}$-B hierarchy, we show that there exist three infinite sets of non-local charges and present a method for constructing them. In section 5, we present briefly an alternate description for such a supersymmetrization which allows the construction of $B$-extensions of systems such as the NLS and the AKNS hierarchies. A brief conclusion is presented in section $\mathbf{6}$. We used the symbolic computer language Reduce [6] and the special package [7] in all calculations presented in this letter.

\section{Model:}

Let us consider a general integrable model of the form

$$
\phi_{t}=(A[\phi])_{x},
$$


where the subscripts refer to differentiation with respect to the corresponding variables. Here, $\phi$ is a general dynamical variable. It can be a purely bosonic function of $x$ alone, in which case, the equation will represent the dynamics of a bosonic integrable system ( $N=0$ supersymmetry). Alternately, $\phi$ may represent a superfield (bosonic or fermionic) depending on $x$ as well as $N$ fermionic coordinates, $\theta_{i}, i=1,2, \cdots, N$, in which case, the dynamical equation will describe an integrable model with $N$-extended supersymmetry. Let us denote the covariant derivatives with respect to the $N$ fermionic coordinates by

$$
D_{i}=\frac{\partial}{\partial \theta_{i}}+\theta_{i} \frac{\partial}{\partial x}, \quad i=1,2, \cdots, N
$$

which satisfy

$$
\left[D_{i}, D_{j}\right]_{+}=\delta_{i j} \frac{\partial}{\partial x}=\delta_{i j} \partial .
$$

We can now introduce a new superfield, $(\phi$ and $\tilde{\phi}$ are superfields depending on the original $N$ fermionic coordinates)

$$
G=G\left(x, \theta_{1}, \cdots, \theta_{N+1}\right)=\tilde{\phi}+\theta_{N+1} \phi,
$$

which depends on one extra fermionic coordinate and has opposite Grassmann parity relative to $\phi$ (the original dynamical variable), and define the dynamical equation

$$
\left(D_{N+1} G\right)_{t}=\left(A\left[\left(D_{N+1} G\right)\right]\right)_{x},
$$

which would represent an integrable system with $(N+1)$-extended supersymmetry. This, therefore, describes the generalization of Beckers' extension to (extended) supersymmetric models. (Basically, the original $\phi$ equation remains unchanged under this extension since $\left.\left(D_{N+1} G\right)\right|_{\theta_{N+1}=0}=\phi$.)

Thus, for example, with $\phi=u(x)$ and $A[u]=u_{x x}+3 u^{2}$, we have the bosonic KdV equation ( $N=0$ supersymmetry) while, with $G=\Phi(x, \theta)$, where $\Phi$ is a fermionic superfield, the equation

$$
\begin{aligned}
(D \Phi)_{t} & =\left(\left(D \Phi_{x x}\right)+3(D \Phi)^{2}\right)_{x}, \\
\text { or, } \quad \Phi_{t} & =\Phi_{x x x}+3 D\left((D \Phi)^{2}\right),
\end{aligned}
$$

represents the $N=1$ supersymmetric KdV-B equation [3]. Similarly, for $\Phi\left(x, \theta_{1}\right)$ a fermionic superfield and $A[\Phi]=-\left(\Phi_{x x}+3 \Phi\left(D_{1} \Phi\right)\right)$, the dynamical equation represents the $N=1$ supersymmetric $\mathrm{KdV}$ equation [⿶], while, with $G\left(x, \theta_{1}, \theta_{2}\right)$ a bosonic superfield, the equation

$$
\begin{aligned}
\left(D_{2} G\right)_{t} & =-\left(\left(D_{2} G_{x x}\right)+3\left(D_{2} G\right)\left(D_{1} D_{2} G\right)\right)_{x}, \\
\text { or, } \quad G_{t} & =-G_{x x x}-3 D_{2}\left(\left(D_{2} G\right)\left(D_{1} D_{2} G\right)\right),
\end{aligned}
$$

would give rise to an $N=2$ extended supersymmetric KdV equation of the $B$-type. Similarly, if $\phi$ represents an $N=2$ superfield and the dynamical equation gives the $N=2$ supersymmetric $\mathrm{KdV}$ equation [8, 9], then, the $G$ equation would correspond to the $N=3$ supersymetric KdV-B equation and so on. While this procedure is quite general, in this letter, we would study the specific model in eq. (6) and bring out properties of this model which are nonetheless common to all such models. We also note here that, as described above, this extension, when applied twice to a given equation, 
would seem to lead to a nonlocal dynamical equation and, therefore, is not useful. Similarly, if the right hand side of eq. (1) is not a total space derivative, this method will also appear to fail. We

would come back to this point in section $\mathbf{5}$, where we would describe an alternate, but equivalent generalization, which can be applied to any given equation and as many times, without introducing non-locality.

\section{Hamiltonians and Hamiltonian Structures:}

Let us next look at the general model of eq. (1). We note that if

$$
H_{n}^{(N)}=\int d x d \theta_{1} \cdots d \theta_{N} h_{n}^{(N)}[\phi], \quad n=1,2, \cdots,
$$

represent the Hamiltonians of the original model, then,

$$
H_{n}^{(N+1)}=\int d x d \theta_{1} \cdots d \theta_{N+1} h_{n}^{(N)}\left[\left(D_{N+1} G\right)\right], \quad n=1,2, \cdots,
$$

would correspond to the Hamiltonians of the extended $B$-model [5]. These are conserved local quantities which would be invariant under the extended supersymmetry and we note that, since the integration, in the second case, is over an additional fermionic variable relative to the definition of the original charges, the Hamiltonians of the new system would have an opposite Grassmann parity compared to those of the original system. It is also not hard to see that the Hamiltonian densities can be written as

$$
h_{n}^{(N)}\left[\left(D_{N+1} G\right)\right]=h_{n}^{(N)}[\phi]+\theta_{N+1} \tilde{h}_{n}^{(N+1)}[\phi, \tilde{\phi}],
$$

so that each of the two parts of the Hamiltonians, namely, the $\theta_{N+1}$ independent term as well as the linear term in $\theta_{N+1}$, will be independently conserved. However, the $\theta_{N+1}$ independent term in the density would give a conserved charge (when integrated over appropriate coordinates) which is invariant only under the lower, $N$-extended supersymmetry.

The Hamiltonian structures of the two systems are also related in a simple manner. Suppose $\mathcal{D}^{(N)}[\phi]$ represents the Hamiltonian structure of the original system so that we can write

$$
\phi_{t}=\mathcal{D}^{(N)}[\phi] \frac{\delta H_{n}^{(N)}[\phi]}{\delta \phi}
$$

Then, it follows that, we can write

$$
\begin{aligned}
\left(D_{N+1} G\right)_{t} & =\mathcal{D}^{(N)}\left[\left(D_{N+1} G\right)\right] \frac{\delta H_{n}^{(N+1)}}{\delta\left(D_{N+1} G\right)}, \\
\text { or, } \quad G_{t} & =\mathcal{D}^{(N+1)}[G] \frac{\delta H_{n}^{(N+1)}}{\delta G}
\end{aligned}
$$


where

$$
\mathcal{D}^{(N+1)}[G]=D_{N+1}^{-1} \mathcal{D}\left[\left(D_{N+1} G\right)\right] D_{N+1}^{-1} .
$$

We note here that the new Hamiltonian structure would have an opposite Grassmann parity from the old one, simply because of the delta functions involving fermionic coordinates. (Such Hamiltonian structures are known as anti-brackets or Buttin brackets [10].) This is consistent with the change of the Grassmann parity for the Hamiltonians that we have already noted. The recursion operators for the two systems are similarly related and, without going into details, we simply note here that

$$
R^{(N+1)}[G]=D_{N+1}^{-1} R^{(N)}\left[\left(D_{N+1} G\right)\right] D_{N+1} .
$$

The Lax description for the two systems are also simply related. For example, we know that the Lax description for the KdV hierarchy is given in terms of the Lax operator of the form

$$
L=\partial^{2}+u,
$$

where $\partial$ represents $\frac{\partial}{\partial x}$. It follows, then, that the Lax operator

$$
L=\partial^{2}+(D \Phi),
$$

would describe the $N=1$ supersymmetric KdV-B hierarchy through the same normal Lax representation,

$$
\frac{\partial L}{\partial t}=\left[L,\left(L^{3 / 2}\right)_{+}\right]
$$

Similarly, the $N=1$ supersymmetric $\mathrm{KdV}$ equation can be described either by a standard representation with the Lax operator [4]

$$
L=\partial^{2}+D_{1} \Phi
$$

or by a Lax operator 11]

$$
L=\partial+D_{1}^{-1} \Phi
$$

with the non-standard Lax representation

$$
\frac{\partial L}{\partial t}=\left[L,\left(L^{3}\right)_{\geq 1}\right]
$$

Correspondingly, the $N=2$ supersymmetric $B$-extension of this system can also have a standard as well as a non-standard representation through the Lax operators

$$
\begin{aligned}
L^{\mathrm{std}} & =\partial^{2}+D_{1}\left(D_{2} G\right), \\
L^{\mathrm{nstd}} & =\partial+D_{1}^{-1}\left(D_{2} G\right) .
\end{aligned}
$$

As opposed to these Lax operators it is also possible to define the Lax operator on the $N=$ 2 superspace. Indeed the method of supercomplexification [12 provides a much more general procedure for obtaining the $B$-extensions and applied to this model, it provides the Lax operator

$$
L^{\mathrm{sc}}=\partial+D_{1}^{-1}\left(D_{2} G\right)-D_{1}^{-1} G_{x} D_{2}^{-1},
$$


which would describe the system with a non-standard Lax representation.

The conserved Hamiltonians of the system can be obtained from the super residues of any of these three Lax operators. Thus, for example, the Hamiltonians of the system can be written in terms of the non-standard Lax operator as

$$
H_{n}=\int d x d \theta_{1} d \theta_{2} \operatorname{Res}\left(L^{\mathrm{nstd}}\right)^{2 n-1}=\int d x d \theta_{1} d \theta_{2} h_{n}
$$

and the first two nontrivial charges of the series have the forms

$$
\begin{aligned}
H_{3} & =\int d x d \theta_{1} d \theta_{2} G\left(D_{1} G_{x}\right) \\
H_{5} & =\int d x d \theta_{1} d \theta_{2}\left[G\left(D_{1} G_{x x x}\right)+4 G\left(D_{1} G_{x}\right)\left(D_{1} D_{2} G\right)\right] .
\end{aligned}
$$

It is worth noting here that the $N=2$ supersymmetric $B$-extension (eq. (6)) has yet another Lax representation [13]. Namely, consider the Lax operator

$$
L=D_{1}+\partial^{-1} D_{2} G-G D_{2} \partial^{-1} .
$$

Then, it is straight forward to check that the non-standard Lax equation

$$
\frac{\partial L}{\partial t}=\left[L,\left(L^{6}\right)_{\geq 1}\right]
$$

gives the $N=2$ equation of eq. (6). However, this Lax operator, surprisingly, does not yield any of the conserved charges of the system. This is indeed a puzzling feature which deserves further study.

\section{Non-local Charges}

Let us now concentrate on the $N=2$ model of the previous section (eq. (6)) for concreteness, although the features we are going to discuss are quite general. We note that although the Hamiltonians for this system are fermionic, as we have discussed, there are also the following bosonic charges which can be explicitly checked to be conserved, namely,

$$
\begin{aligned}
\tilde{H}_{1} & =\int d x d \theta_{1} d \theta_{2} G, \\
\tilde{H}_{2} & =\int d x d \theta d \theta_{2} G^{2}, \\
\tilde{H}_{3} & =\int d x d \theta_{1} d \theta_{2}\left(\frac{1}{3} G^{3}-G\left(D_{1} D_{2} G\right)\right) .
\end{aligned}
$$


At first sight, the existence of such charges with opposite Grassmann parity would seem surprising and, in fact, the existence of such a charge was already noted earlier in connection with the $N=1$ supersymmetric KdV-B system and was termed "exotic" [5]. In what follows, we would try to clarify the origin of such charges and show that, in such systems, there are, at least, two infinite sets of conserved non-local charges (and may be more) and that these "exotic" charges are part of an infinite hierarchy of non-local charges.

To begin with, let us recall that supersymmetric integrable systems, in general, possess nonlocal charges [14, 15, 16, 11]. However, in the study of dispersionless limits of $B$-extended models, it was already noted 17] that there are two sets of conserved, non-local charges present. In fact, a little bit of analysis shows that the $B$-extensions of integrable models will always have at least two infinite sets of non-local conserved charges. For example, in the $N=2$ supersymmetric KdV-B hierarchy, let us note that the charges

$$
\bar{H}_{n}=\int d x d \theta_{1} d \theta_{2}\left(D_{2}^{-1} \operatorname{ses}\left(L^{\mathrm{nstd}}\right)^{2 n-1}\right)=\int d x d \theta_{1} d \theta_{2}\left(D_{2}^{-1} h_{n}\right),
$$

will be conserved simply because these correspond to the conserved charges of the original system and the original equations are unmodified by this extension. Such a hierarchy of charges will always be present. (In the spirit of eq. (9), these charges would be obtained from the $\theta_{2}$ independent part of the densities.) They are manifestly non-local and, consequently, are not invariant under the $N=2$ extended supersymmetry. Let us also note that, by definition,

$$
\int d x d \theta_{1} d \theta_{2}\left(\partial^{-1} D_{1} D_{2} s \operatorname{Res}\left(L^{n s t d}\right)^{2 n-1}\right)=0
$$

There is also a second set of non-local charges which one can construct. Namely, let us evaluate the square root of the Lax operator for the non-standard representation. Conventionally, the super residues of the odd powers of the square root of the Lax operator gives rise to conserved nonlocal charges in supersymmetric integrable models. As we will now show, the system under study presents a novel feature and, consequently, leads to new charges. Let us note that since both $D_{1}$ and $D_{2}$ satisfy

$$
D_{1}^{2}=\partial=D_{2}^{2},
$$

it follows that the general form of the square root can be determined to have the form

$$
\begin{aligned}
\left(L^{\mathrm{nstd}}\right)^{1 / 2}= & \alpha D_{1}+\beta D_{2}+2 \alpha\left(D_{2}^{-1} G\right)-\left(\alpha\left(\partial^{-1} D_{1} D_{2} G\right)+\beta G\right) D_{1}^{-1} \\
& +\left(\alpha\left(D_{2} G\right)-\beta\left(D_{1} G\right)-\beta\left(D_{2}^{-1} G^{2}\right)\right) \partial^{-1} \\
& +\frac{1}{2}\left(\alpha\left(\partial^{-1} D_{1} D_{2} G\right)^{2}+\beta\left(\partial^{-1} D_{1} D_{2} G^{2}\right)\right) D_{1}^{-3}+\cdots
\end{aligned}
$$

where the constant parameters $\alpha$ and $\beta$ are constrained to satisfy $\alpha^{2}+\beta^{2}=1$. While non-local charges have been constructed earlier from square (and quartic) roots [15, 11, 16], here we have the novel feature that there is a one parameter family of square roots of the Lax operator. We think this feature would exist in extended supersymmetric models with $N>1$. In fact, let us note that for $\alpha=1$ and $\beta=0$, this square root coincides with what has been calculated earlier [11, 16]. But, this is, in fact, the more general form of the square root with a richer structure. 
From the structure of this general square root, let us note that we can construct conserved charges by taking the "sRes" of odd powers of the square root and would, in general, give nonlocal charges. In fact, let us note that the first few of these charges have the forms $\left(d z=d x d \theta_{1} d \theta_{2}\right)$

$$
\begin{aligned}
\int d z \operatorname{sen}\left(L^{\mathrm{nstd}}\right)^{1 / 2}= & -\beta \int d z G \\
\int d z \operatorname{sen}\left(L^{\mathrm{nstd}}\right)^{3 / 2}= & -\frac{3 \alpha}{2} \int d z G^{2} \\
\int d z \operatorname{sen}\left(L^{\mathrm{nstd}}\right)^{5 / 2}= & \int d z\left[2 \beta\left(\frac{1}{3} G^{3}-G\left(D_{1} D_{2} G\right)\right)+\beta\left(D_{2}^{-1}\left(G_{x}\left(D_{1} G\right)\right)\right)\right. \\
& \left.-\frac{\alpha}{2}\left(\frac{1}{3}\left(D_{1}^{-1} D_{2} G\right)^{3}+2\left(D_{1}^{-1}\left(\left(D_{2} G\right)\left(D_{1} D_{2} G\right)\right)\right)\right)\right] .
\end{aligned}
$$

Thus, we see that the "sRes" of the odd powers of the square root leads to conserved charges which are a combination of new non-local charges (the first few of which are local) as well as old ones of the form eq. (20). In fact, if we neglect the old non-local charges in these expressions, we see that the one parameter family of charges really leads to two distinct sets of conserved charges. Thus, for example, when $\alpha=1$ (and, therefore, $\beta=0$ ), the non-local charges coincide with what has been obtained earlier [15, 11, 16]. However, when $\beta=1$, we have a new set of non-local conserved charges for the system. Thus, we conclude that, in this $N=2$ supersymmetric model, we have, in fact, three sets of conserved non-local charges. Furthermore, we now recognize that the three "exotic" charges belong to this hierarchy of non-local charges and can only be obtained if we take the general square root. (In other words, the first few members of the non-local hierarchy of charges is really local, even though higher order ones are truly non-local. We have explicitly verified with REDUCE that there are no more local "exotic" (bosonic) conserved charges present.)

To complete the story of the "exotic" charges, let us look at the simpler system of $N=1$ supersymmetric KdV-B hierarchy. Here the Lax operator, as we have seen in eq. (14), has the form

$$
L=\partial^{2}+(D \Phi)
$$

The fermionic Hamiltonians of the system are given by

$$
H_{n}=\int d x d \theta \operatorname{Res}\left(L^{(2 n-1) / 2}\right)=\int d x d \theta h_{n}, \quad n=1,2, \cdots,
$$

and the first set of non-local charges are given by

$$
\bar{H}_{n}=\int d x d \theta\left(D^{-1} \operatorname{Res}\left(L^{(2 n-1) / 2}\right)\right)=\int d x d \theta\left(D^{-1} h_{n}\right) .
$$

Since, in this case, we have only one fermionic coordinate, the quartic root is without any arbitrary parameter and the residues of the odd powers of it give rise to a linear combination of new non-local conserved charges and the ones in eq. (24). Ignoring these old charges, we can write the new set of non-local charges to be coming from

$$
\tilde{H}_{n}=\int d x d \theta \operatorname{Res}\left(L^{(2 n-1) / 4}\right),
$$


These are bosonic charges and explicitly, the first few of them have the form

$$
\begin{aligned}
\tilde{H}_{1} & =\frac{1}{2} \int d x d \theta \Phi \\
\tilde{H}_{2} & =\frac{1}{4} \int d x d \theta\left(D^{2} \Phi\right)=0 \\
\tilde{H}_{3} & =\frac{1}{4} \int d x d \theta \Phi(D \Phi) \\
\tilde{H}_{4} & =\frac{3}{8} \int d x d \theta \Phi\left(D^{3} \Phi\right) \\
\tilde{H}_{5} & =\frac{1}{8} \int d x d \theta \Phi\left(\left(D^{5} \Phi\right)+2(D \Phi)^{2}\right) .
\end{aligned}
$$

Of these, only $\tilde{H}_{3}$ was found earlier and termed "exotic" [5]. We see that it belongs to a hierarchy of non-local charges, the first four of which are, in fact, local. (We suspect that, in this particular case, this new set of charges is indeed local. This follows from an analysis of the structure of the charges in the dispersionless limit [17]. However, this is not a general feature.)

\section{Alternate description:}

As we had noted earlier, the conventional $B$-extension cannot be applied to equations where the time evolution of the dynamical variable is not a space derivative. Furthermore, even when the $B$-extension exists, it gives non-local equations if applied more than once. In this section, we will describe very briefly, an alternate extension which does not suffer from this problem. Let us consider an integrable system of the form

$$
\phi_{t}=B[\phi] .
$$

If we now define a superfield

$$
\bar{G}\left(x, \theta_{1}, \cdots, \theta_{N+1}\right)=\phi+\theta_{N+1} \bar{\phi},
$$

then, we note that the new superfield depends on one extra fermionic coordinate and has the same Grassmann parity as the original variable $\phi$. If we now define a dynamical system described by

$$
\bar{G}_{t}=B[\bar{G}] \text {. }
$$

then, this system would be integrable. We note that the $\theta_{N+1}$ independent part of this equation

would correspond to eq. (27), the original equation, so that it provides an alternate description of the $B$-extension. In fact, when we can write $B[\phi]=(A[\phi])_{x}$, the two descriptions would be equivalent and will map into each other under

$$
\bar{G} \rightarrow\left(D_{N+1} G\right) .
$$

All the discussions of the earlier sections can be carried out in this framework as well. However, the advantage of such a description may lie in the fact that the $B$-extended equation, in eq. (29), 
is exactly like the original equation independent of whether the right hand side is a total derivative or not. Consequently, we can apply $B$-extension to any given equation and as many times without running into the problems of non-locality. (In simple terms, the new variables in the alternate representation are more local than the older ones.) As a result, systems, such as the NLS and the AKNS hierarchies, which were thought not to have a local $B$-extension (in the standard approach) [18, 19], can actually have one in this alternate description.

\section{Conclusion}

In this letter, we have studied systematically the general features of $B$-extension of any given integrable system. We have brought out general features such as the Hamiltonians, Hamiltonian structures and recursion operators. We have clarified the origin of "exotic" charges in such models and have identified them as belonging to an infinite set of non-local charges. We have shown that, in such models, there naturally exist, at least, two infinite sets of non-local charges and, for $N>1$ supersymmetry, even more. We have explicitly shown that the $N=2$ supersymmetric KdV-B hierarchy has three sets of non-local conserved quantities and have discussed their construction starting from the Lax operator.

\section{Acknowledgments}

A.D. acknowledges support in part by the U.S. Dept. of Energy Grant DE-FG 02-91ER40685 while Z.P. is supported in part by the Polish KBN Grant 2 P0 3B 13616.

\section{References}

[1] G.B. Whitham, Linear and Nonlinear Waves, John Wiley, 1974; L.D. Faddeev and L.A. Takhtajan, Hamiltonian Methods and the Theory of Solitons, Springer, 1987; A. Das, Integrable Models, World Scientific, 1989.

[2] D.J. Gross and A.A. Migdal, Phys. Rev. Lett. 64 (1990) 127; D.J. Gross and A.A. Migdal, Nucl. Phys. B340 (1990)333; E. Brézin and V.A. Kazakov, Phys. Lett. B236 (1990) 144; M. Douglass and S.H. Shenker, Nuc. Phys. B335 (1990) 635.

[3] K. Becker and M. Becker, Mod. Phys. Lett. A8 (1993) 1205.

[4] Yu. I. Manin and A. O. Radul, Comm. Math. Phys. 98 (1985) 65; P. Mathieu, Phys. Lett. B203 (1988) 287; P. Mathieu, J. Math. Phys. 29 (1988) 2499. 
[5] J.M. Figueroa-O'Farrill and S. Stanciu, Phys. Lett. B316 (1993) 282.

[6] A. Hearn Reduce User's Manual 3.7, (1999).

[7] Z. Popowicz Compt. Phys. Commun 100 (1997) 277.

[8] P. Laberge and P. Mathieu Phys. Lett B215 (1988) 718; P. Labelle and P. Mathieu J. Math. Phys. 32 (1991) 923.

[9] Z. Popowicz J. Phys. A29 (1996) 4987; Z. Popowicz Phys. Lett A174 (1993) 411.

[10] D.A. Leites, Dokl. Akad. Nauka SSSR 236 (1977) 804 (in Russian); A. Batalin and G.A. Vilkovsky, Phys. Lett. B102 (1981) 27; A. Batalin and G.A. Vilkovsky, Nuc. Phys. B234 (1984) 106.

[11] J.C. Brunelli and A. Das, Phys. Lett. B337 (1994) 303; J.C. Brunelli and A. Das, Int. J. Mod. Phys. A10 (1995) 4563.

[12] Z. Popowicz Phys. Lett. B459 (1999) 150.

[13] F. Delduc, L. Gallot and A. Sorin Nucl. Phys B558 (1999) 535.

[14] P.H.M. Kersten, Phys. Lett. A134 (1988) 25.

[15] P. Dargis and P. Mathieu, Phys. Lett. A176 (1993) 67.

[16] J.C. Brunelli and A. Das, Phys. Lett. B354 (1995) 307.

[17] J. Barcelos-Neto, A. Constandache and A. Das, Phys. Lett. A268 (2000) 342.

[18] J.C. Brunelli and A. Das, Phys. Lett. B409 (1997) 229.

[19] H.A. Aratyn and A. Das, Mod. Phys. Lett. A15 (1998) 1185. 